\title{
Alterações da Hemostasia em Crianças Submetidas a Cirurgia Cardíaca com Circulação Extracorpórea
}

\author{
Fernanda Maria Rebouças da Costa Silveira, Dayse Maria Lourenço, Miguel Maluf, \\ Werther B. Carvalho, Enio Buffolo, Antonio Carlos Carvalho
}

São Paulo, SP

\begin{abstract}
Objetivo - Avaliar as alterações de hemostasia encontradas em crianças submetidas a cirurgia cardíaca com circulação extracorpórea (CEC).

Métodos - Estudamos 17 crianças no pré e pós-operatório $(\mathrm{PO})$ imediato, no $1^{\circ} \mathrm{PO}$ e entre o $4^{\circ}$ e $7^{\circ} \mathrm{PO}$, analisando o hematócrito, tempo de protrombina $(T P)$, tempo de tromboplastina parcial ativado, fibrinogênio, contagem de plaquetas, fator $V$ e tempo de lise de euglobulina (TLE). Os pacientes foram divididos em grupos com e sem sangramento excessivo no $P O$.

Resultados - Houve alterações significantes no intraoperatório com aumento do TP e tempo de tromboplastina parcial ativado e redução do fibrinogênio, fator $V$ e do TLE. Seis (35\%) crianças sangraram excessivamente. A contagem de plaquetas foi significantemente menor no intra-operatório; neste grupo o tempo de CEC foi maior.

Conclusão - Alterações de hemostasia no intra e PO ocorrem como conseqüência da ativação da cascata de coagulação e fibrinólise. A incidência de sangramento excessivo é alta nesta faixa etária. Os grupos com e sem sangramento excessivo diferenciaram-se pelo maior tempo de CEC e contagem reduzida de plaquetas.
\end{abstract}

Palavras-chave: hemostasia, cirurgia cardíaca, circulação extracorpórea

\section{Hemostatic Changes in Children Undergoing Heart Surgery with Cardiopulmonary Bypass}

Purpose - We evaluated hemostasic changes in children undergoing open heart surgery with cardiopulmonary bypass $(C P B)$.

Methods - We studied 17 children before, during surgery, in the immediate, first and between the $4^{\text {th }}$ and $7^{\text {th }}$ postoperative days, measuring hematocrit, prothrombin time, activated partial thromboplastin time, fibrinogen, platelet count, factor V and euglobulin lysis time. Children were divided in those with and without excessive bleeding in the postoperative period.

Results - We observed significant prolongation of prothrombin time and activated partial thromboplastin time, reduction of fibrinogen and factor $V$, and shortening of euglobulin lysis time. Six (35\%) children bled excessively. Platelet count reduction was greater in the intra operative period in these cases and the duration of $C P B$ was longer in this group.

Conclusion - Changes in hemostasis during open heart surgery are due to coagulation cascade disorders as well as fibrinolysis. The incidence of excessive bleeding is higher in the pediatric group. Prolonged CPB time and greater reduction in platelet count differentiated both groups.

Key-words: hemostasis, heart surgery, cardiopulmonary bypass

Arq Bras Cardiol, volume 70 (nº 1), 29-35, 1998

A cirurgia cardíaca com circulação extracorpórea (CEC) promove uma série de alterações na hemostasia e foram realizado estudos para avaliar a incidência e o tipo de

Universidade Federal de São Paulo - Escola Paulista de Medicina Correspondência: Antonio Carlos Carvalho - Av. Jandira, 731/23 - 04080-004 São Paulo, SP

Recebido para publicação em 20/2/97

Aceito em 30/10/97 distúrbio envolvido, além de suas repercussões clínicas, associação com componentes da máquina de CEC e tipos de procedimentos cirúrgicos ${ }^{1}$.

Por ocasião da cirurgia cardíaca com CEC, desencadeia-se ativação da coagulação, que é bloqueada pelo uso da heparina. As complicações que podem surgir em relação a sangramento excessivo dependem da inadequação dos mecanismos da coagulação e fibrinólise ou decorrem da utilização do anticoagulante. Além disso, o sangramento 
pode ser decorrente da própria técnica cirúrgica. Durante a CEC, ocorre hemólise de graus variáveis; nas perfusões mais prolongadas, a hemólise pode ser maciça ${ }^{2}$.

As plaquetas aderem à superfície dos circuitos, formando microagregados, sendo, portanto, consumidas. Em relação aos fatores da coagulação, há redução dos mesmos, devido ao consumo e à hemodiluição ${ }^{3,4}$. Manifestações trombóticas são menos freqüentes que as hemorrágicas e mais encontradas em pacientes cianóticos e nos portadores de prótese valvar ${ }^{5,6}$.

A utilização de CEC pode se associar a hemorragias graves no pós-operatório (PO). Em diferentes estudos, a incidência varia de 5 a $25 \%$ e, além das causas já citadas, temos rebote de heparina, excesso de protamina e defeitos qualitativos na formação do polímero de fibrina. A coagulação intravascular disseminada é tida como causa rara. $\mathrm{Na}$ verdade, observa-se que o sangramento excessivo no intra-operatório (IO) dificilmente é devido a uma única causa $^{7,8}$.

Os estudos da literatura no tocante a hemostasia, em sua maior parte, foram feitos em adultos, ou em grupos mistos de crianças e adultos. O objetivo do presente trabalho foi medir parâmetros da hemostasia, somente em crianças, que foram submetidas à cirurgia cardíaca com CEC.

\section{Métodos}

Foram analisados os dados referentes à evolução de 17 crianças que se submeteram a cirurgia cardíaca eletiva. Na época da realização do estudo, cerca de 100 crianças foram submetidas a cirurgia cardíaca com e sem utilização de CEC. Outros protocolos de pesquisa estavam sendo desenvolvidos naquele período, o que impossibilitava que todas as crianças participassem de mais de um protocolo, devido à grande espoliação de sangue para realização dos exames laboratoriais. Assim, a inclusão foi feita obedecendo-se uma sequiência pré-estabelecida dos protocolos a serem de- senvolvidos, que não era baseada nas características dos pacientes, mas sim na disponibilidade dos pesquisadores participantes. Neste contexto, a seleção dos pacientes foi consecutiva, embora não incluísse todas as crianças da programação cirúrgica da disciplina. $\mathrm{O}$ estudo foi prospectivo e a análise dos dados foi feita após a inclusão de todas as crianças.

O critério de exclusão foi o caráter de urgência da cirurgia, devido à dificuldade de realização dos exames pelo Laboratório da Hematologia, fora do horário de rotina, bem como pela impossibilidade de acompanhamento pelo pesquisador. Excluiu-se, também, crianças no período neonatal, devido às peculiaridades do sistema de hemostasia, nessa faixa etária.

A idade variou de 7 a 128 (mediana de 33) meses. Dez crianças eram do sexo feminino e sete do masculino. Cinco (29\%) eram portadoras de cardiopatias cianogênicas, enquanto $12(71 \%)$ eram portadoras de cardiopatias acianogênicas. A distribuição quanto à idade e à cardiopatia de base estão representadas na figura 1 .

Foram registradas as intercorrências eventuais, o tempo de anóxia e de perfusão, o tipo de oxigenador e medicamentos utilizados. No PO foram anotados os débito dos drenos e a reposição de hemoderivados.

O estado nutricional foi avaliado pelo critério de Gomez para crianças com idade $<5 \operatorname{anos}^{9}$ e de Waterlow para crianças com idade $>5$ anos ${ }^{10}$.

O protocolo de cirurgia cardíaca com CEC para crianças, da Disciplina de Cirurgia Cardiovascular do Departamento de Cirurgia da UNIFESP-EPM foi adotado em todos os casos ${ }^{11}$. Foi utilizado nas perfusões um oxigenador de bolhas do tipo Macchi. O perfusato foi constituído de solução cristalóide acrescida de sangue total, plasma ou concentrado de hemácias. A dose total utilizada de heparina variou de 3,9 a $7 \mathrm{mg}$ por quilo de peso. Ao final da perfusão, todas as crianças receberam cloridrato de protamina na proporção de 1:0,8 a 1:1,5 com relação à heparina.

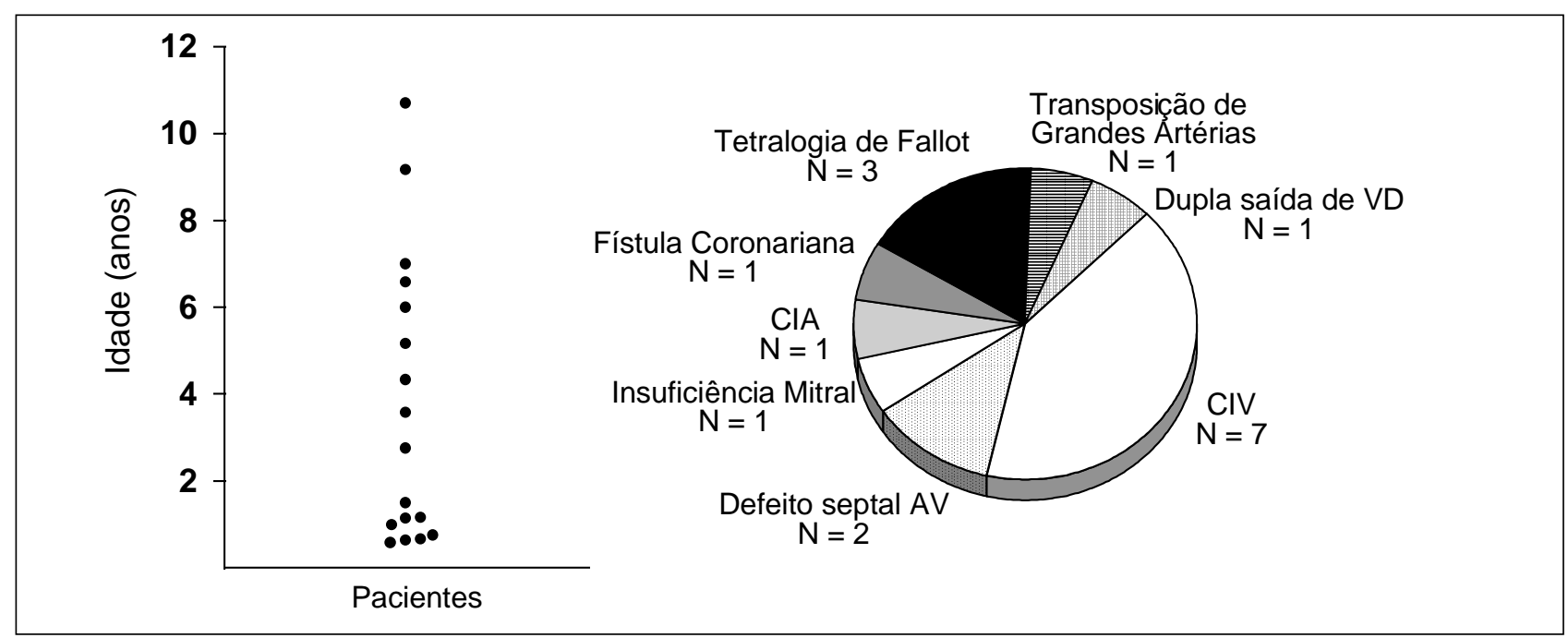

Fig. 1 - Distribuição dos 17 pacientes quanto à idade (em anos) e a cardiopatia de base (CIV- comunicação interventricular, AV-atrioventricular, CIA- comunicação interatrial, VDventrículo direito). 


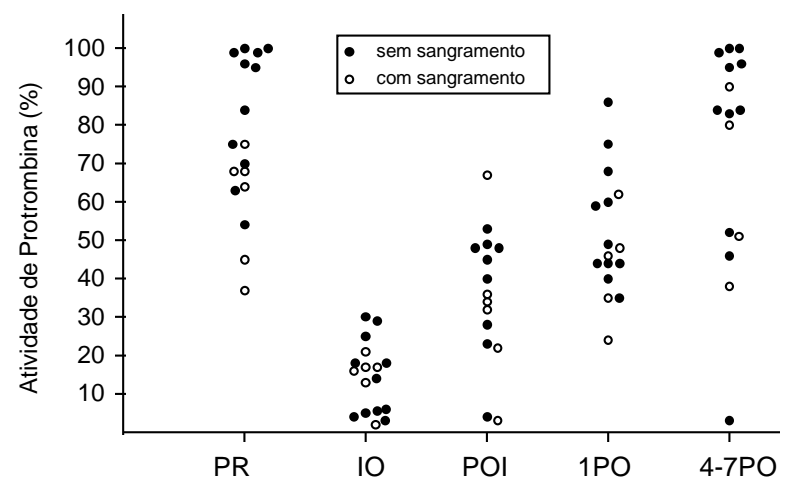

A

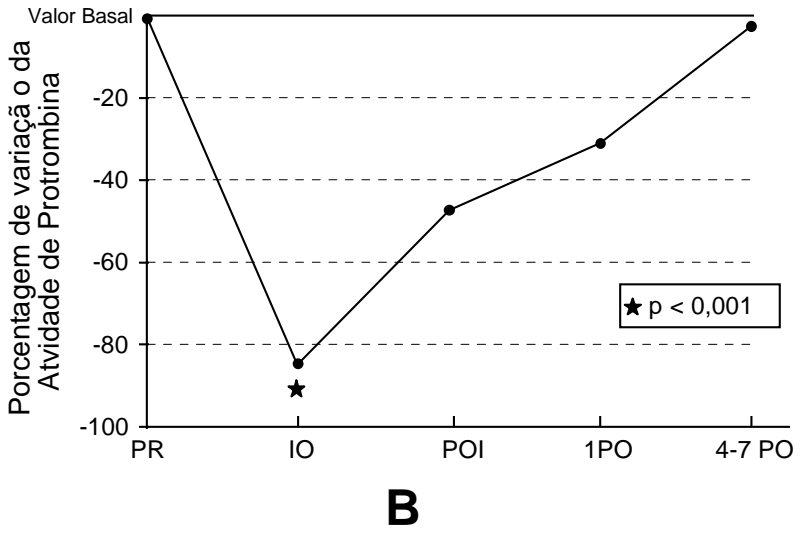

Fig. 2 - Valores (gráfico A) e médias das variações (gráfico B) da atividade de protrombina nos tempos: pré-operatório (PRÉ), intra-operatório (IO), pós-operatório imediato (POI), $1^{\circ}$ dia de pós-operatório (1PO) e entre o $4^{\circ}$ e $7^{\circ}$ dias de pós-operatório (4-7PO) para pacientes com e sem sangramento excessivo.

As amostras de sangue foram colhidas nos seguintes tempos: pré-operatório (PRÉ), geralmente no dia anterior à cirurgia; no IO durante a CEC; no pós-operatório imediato (POI), cerca de $1 \mathrm{a} 2 \mathrm{~h}$ após chegada à UTI; no $1^{\circ}$ dia de PO (1PO) e no final, entre o $4^{\circ}$ e $7^{\circ}$ dias de PO (4-7PO).

O sangue total foi colhido em citrato de sódio $0,1 \mathrm{M}$ na proporção de uma parte de anticoagulante para nove partes de sangue (v/v). O plasma pobre em plaquetas (PPP) foi obtido após centrifugação a $3000 \mathrm{rpm}$ por $15 \mathrm{~min}$. O tempo de protrombina (TP), o tempo de tromboplastina parcial ativado (TTPA) e a contagem de plaquetas foram realizados, imediatamente, e os demais testes foram realizados em ensaio posterior, a partir das alíquotas de PPP congeladas $\mathrm{a}-70^{\circ} \mathrm{C}$.

OTP foi realizado segundo o método de Quick, usando tromboplastina de cérebro de coelho ${ }^{12}$ e o resultado foi expresso em atividade de protrombina (AP), derivada de curva-padrão construída com plasma controle normal. OTTPA foi feito usando-se cefalina de cérebro humano e caolim, como ativador da fase de contato ${ }^{13}$. A contagem de plaquetas foi realizada em sangue total citratado, em câmara de Neubauer, utilizando-se um microscópio de fase ${ }^{14}$. A dosagem do fator $\mathrm{V}$ foi feita com plasma, artificialmente, deficiente em fator $\mathrm{V}$, segundo a técnica de Quick ${ }^{15}$. O fibrinogênio foi medido pelo método de Clauss ${ }^{16}$. O tempo de lise de euglobulina (TLE) foi realizado pelo método de Kluft ${ }^{17}$. O hematócrito foi determinado em sangue total colhido em EDTA, utilizando-se contador eletrônico Coulter ${ }^{\circledR}$, modelo SSR.

A evolução dos testes laboratoriais nos diversos tempos foi estudada através da análise de variância por postos de Friedman. Quando houve diferença significante, a análise foi complementada pelo teste de comparações múltiplas. Os grupos com e sem sangramento excessivo foram comparados através do teste de MannWhitney. Foi usado o teste t de Student para grupos independentes para comparar pacientes com e sem sangramento excessivo em relação ao tempo de CEC. O nível de significância adotado foi de $5 \%{ }^{18}$.
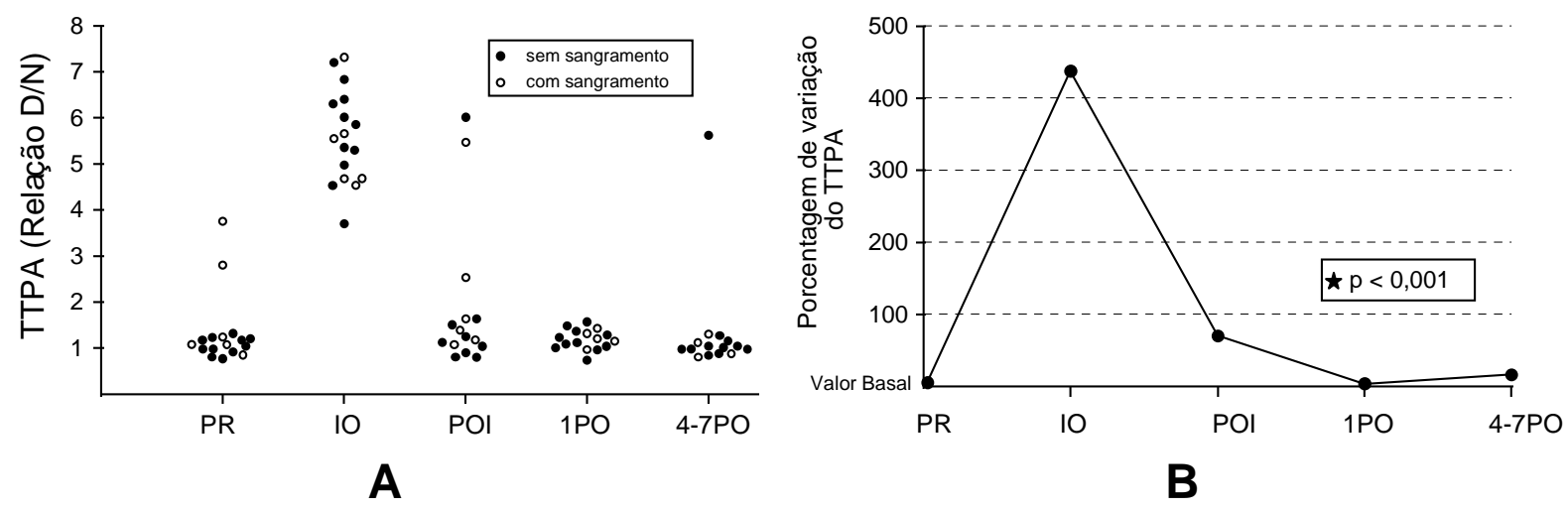

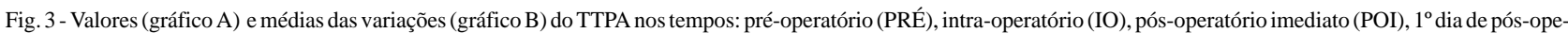
ratório (1PO) e entre o $4^{\circ}$ e $7^{\circ}$ dias de pós-operatório (4-7PO) para pacientes com e sem sangramento excessivo. 


\section{Resultados}

A análise do estado nutricional mostrou que 10 das 11 crianças $<5$ anos eram desnutridas e apenas uma era eutrófica. Segundo os critérios de Gomez, duas tinham desnutrição de $1^{\circ}$ grau, cinco de $2^{\circ}$ e três de $3^{\circ}$. Entre as seis crianças com mais de cinco anos, os critérios de Waterlow mostraram haver uma com sobrepeso, uma com desnutrição pregressa, três com desnutrição atual e uma com desnutrição crônica.

Na avaliação PRÉ, seis (35\%) crianças apresentaram contagem de plaquetas inferior a $150.000 / \mathrm{mm}^{3}$, sendo três portadoras de cardiopatias congênitas cianogênicas. Nenhum paciente tinha hematócrito $<30 \%$; duas crianças apresentaram TP $<60 \%$ e duas crianças tinham TTPA prolongado.

Os pacientes foram divididos em dois grupos: com e sem sangramento excessivo. Os critérios adotados para se caracterizar sangramento excessivo foram: a) sangramento por dreno de mediastino, acima de $3 \mathrm{ml} / \mathrm{kg} / \mathrm{h}$ nas primeiras 6h; b) sangramento súbito em grande quantidade por drenos pleural ou de mediastino; c) sangramento difuso incluindo hemorragia pulmonar, digestiva ou hematúria.

Seis crianças sangraram, excessivamente, sendo que

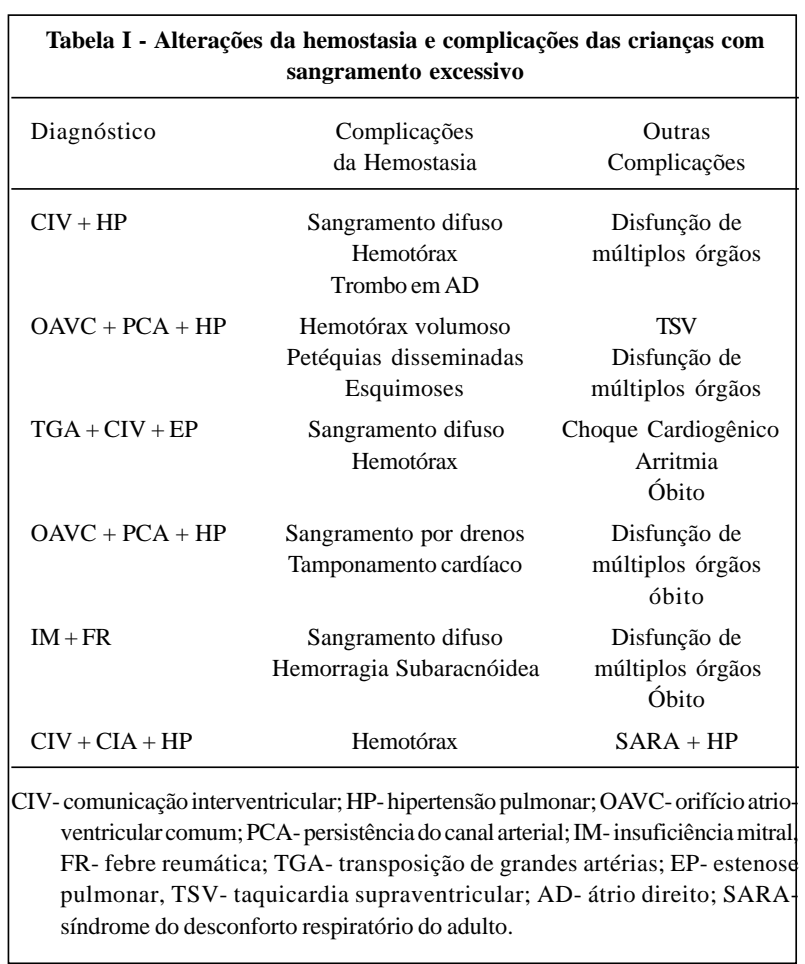

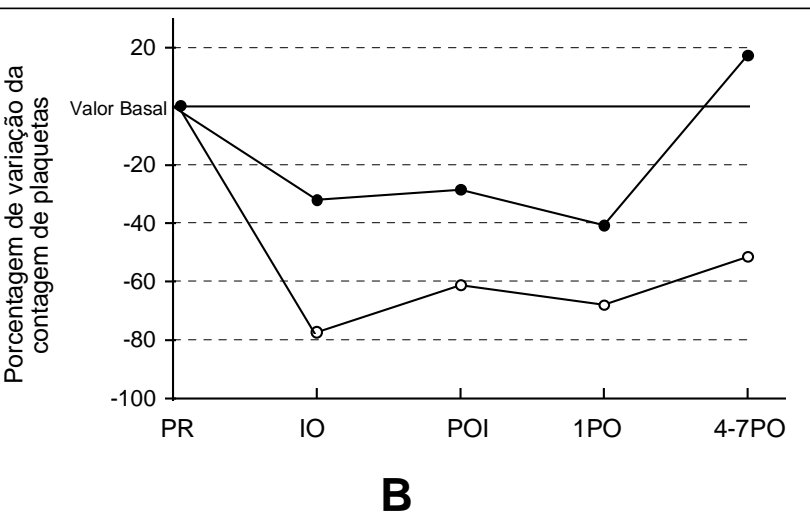

Fig. 4 - Valores (gráfico A) e médias das variações (gráfico B) da contagem de plaquetas nos tempos: pré-operatório (PRÉ), intra-operatório (IO), pós-operatório imediato (POI), $1^{\circ}$ dia de pós-operatório (1PO) e entre o $4^{\circ} \mathrm{e} 7^{\circ}$ dias de pós-operatório (4-7PO) para pacientes com e sem sangramento excessivo.
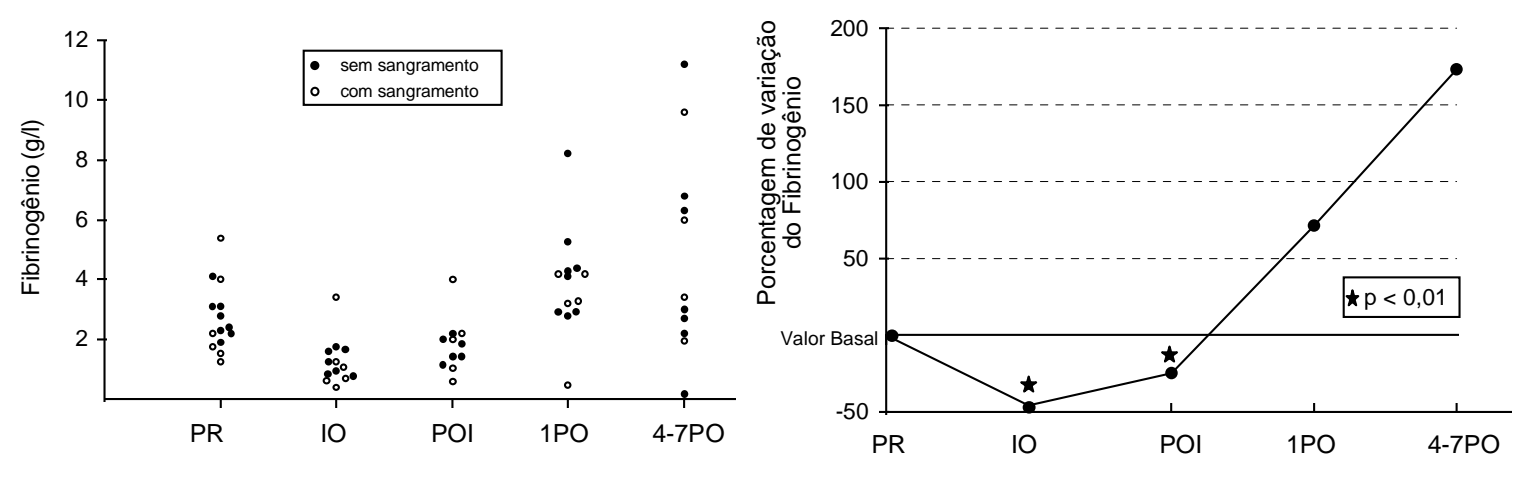

A

B

Fig. 5 - Valores (gráfico A) e médias das variações (gráfico B) do fibrinogênio nos tempos: pré-operatório (PRÉ), intra-operatório (IO), pós-operatório imediato (POI), $1^{\circ}$ dia de pósoperatório (1PO) e entre o $4^{\circ} \mathrm{e} 7^{\circ}$ dias de pós-operatório (4-7PO) para pacientes com e sem sangramento excessivo. 


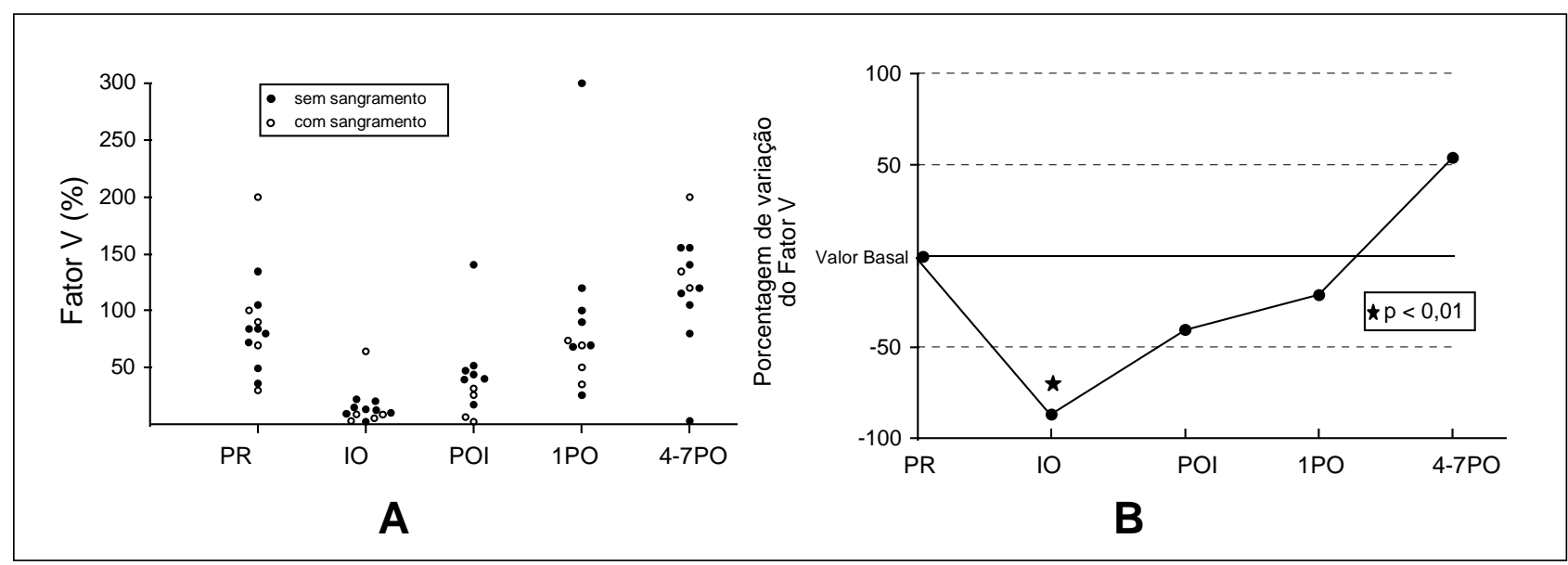

Fig. 6 - Valores (gráfico A) e médias das variações (gráfico B) do fator V nos tempos: pré-operatório (PRÉ), intra-operatório(IO), pós-operatório imediato (POI), $1^{\circ}$ dia de pós-operatório (1PO) e entre o $4^{\circ}$ e $7^{\circ}$ dias de pós-operatório (4-7PO) para pacientes com e sem sangramento excessivo.

quatro delas apresentaram disfunção de múltiplos órgãos e sistemas (tab. I). Uma criança foi reoperada devido a sangramento por ruptura de parede de tronco pulmonar e apresentava alteração da coagulação. Uma criança desenvolveu trombo em átrio direito, que se desfez sem uso de trombolíticos. O tempo de perfusão na CEC variou de 38 a 130

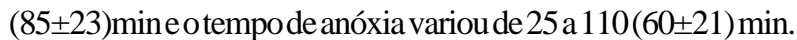
Houve diferença significante (teste t de Student, $\mathrm{p}<0,05$ ) quanto ao tempo de CEC entre os grupos com sangramento (média de 102min) e sem sangramento (média de 76min).

Exceto para a contagem de plaquetas, houve alteração estatisticamente significante dos testes da coagulação no IO, pela análise de variância por postos de Friedman. Não houve diferença significante na variação dos testes no grupo de crianças com e sem sangramento excessivo (MannWhitney, $\mathrm{p}>0,05)$.

A queda de hematócrito observada no IO foi de $19 \%$ com relação ao valor basal do PRÉ $(\mathrm{p}<0,05)$ e já estava corrigida no POI, com redução de $7 \%$ em relação ao valor basal. No 1 PO o hematócrito estava $2 \%$ abaixo do basal e, entre o 4-7PO, 4\% acima do valor basal.

Houve redução de $85 \%$ da AP no IO (p<0,05) e de $47 \%$ no POI, com recuperação parcial no $1 \mathrm{PO}$, quando a redução foi de $31 \%$ do valor basal. No 4-7PO, o AP estava 3\% abaixo do valor basal (fig. 2).

Houve prolongamento significante do TTPA no IO (438\%). No POI, o prolongamento foi de $71 \%$, normalizandose já no 1 PO (4\%) e voltando a se prolongar no 4-7PO (17\%), mas não de modo significante (fig. 3).

A redução da contagem de plaquetas no IO (46\%) não foi significante para as 17 crianças. Entretanto, quando analisamos separadamente os grupos com e sem sangramento excessivo, observamos maior redução no grupo com sangramento no IO (Mann-Whitney, $\mathrm{p}<0,05)$. No POI, a redução da contagem de plaquetas foi de $29 \%$ para as que não sangraram e de $61 \%$ para as que sangraram. No 1PO, os valores foram, respectivamente, $41 \%$ e $68 \%$. No 4-7PO a contagem de plaquetas foi normal para as crianças sem sangramento (17\% acima do valor basal) e se situava ainda a $52 \%$ do valor basal para as que tiveram sangramento excessivo (fig. 4).

Houve redução de $46 \%$ do valor basal do fibrinogênio no IO (Friedman, p<0,05). No POI o fibrinogênio estava $25 \%$ abaixo do valor basal e a partir do 1PO houve aumento significante (72\%), mantido até no 4-7PO (173\%). A evolução dos níveis do fibrinogênio está representada na figura 5.

Observou-se redução estatisticamente significante dos níveis de fator $\mathrm{V}$ no IO (87\%). No POI e no 1PO, os valores estavam $41 \%$ e $21 \%$ abaixo do valor basal, respectivamente. Assim como para o fibrinogênio, houve aumento do fator $\mathrm{V}$ a 54\% do valor basal no 4-7PO (fig. 6).

Ocorreu encurtamento significante do TLE IO (74\% abaixo do valor basal) indicando ativação da fibrinólise, que se recuperou já no POI para valores cerca de $20 \%$ acima do valor basal.

\section{Discussão}

A porcentagem de crianças desnutridas é, sem dúvida, muito alta (88\%). Uma vez que os fatores de coagulação são proteínas, isto pode ter influência desfavorável no perfil de coagulação. Na bibliografia revisada, em relação a complicações hematológicas, não houve referências a possível influência da situação nutricional, provavelmente, pelo melhor estado nutricional dos pacientes referidos na literatura internacional. Em estudo realizado neste mesmo serviço, Leite e $\operatorname{col}^{19}$ encontraram prevalência de $78 \%$ de desnutrição entre 40 crianças submetidas a cirurgia cardíaca, mas os autores não avaliaram sua influência sobre sangramento. Nas crianças trombocitopênicas, no PRÉ, não se pôde estabelecer correlação com a existência de cardiopatia cianogênica, como referido por outros autores ${ }^{2,20,21}$, provavelmente pela pequena amostra de pacientes cianóticos $(\mathrm{N}=5)$.

As diferenças relacionadas à perfusão foram mínimas, já que princípios semelhantes de hemodiluição, anticoagulação e hipotermia foram adotados. Excetuando-se a contagem de plaquetas, houve alteração significante dos demais parâmetros da hemostasia. Houve redução signi- 
ficante da AP, da atividade do fator $\mathrm{V}$ e do fibrinogênio, aliados ao aumento do TTPA e encurtamento do TLE. Apesar da queda de contagem de plaquetas não ter apresentado significado estatístico, houve uma redução considerável para quase metade dos níveis pré-operatórios. O conjunto destes achados sugere ter havido ativação da coagulação e da fibrinólise.

O TTPA é um teste bastante sensível à ação de heparina e obtivemos no IO um aumento em média de 4,4 vezes o valor basal. Sem dúvida, pudemos observar uma heparinização efetiva, mas dados da influência in vitro da heparina sobre a AP e o fibrinogênio sugerem que, possivelmente, houve consumo dos fatores de coagulação durante a CEC, uma vez que níveis altos de heparina in vitro não foram capazes de modificar esses parâmetros (dados não mostrados).

Não se observou influência marcante da hemodiluição sobre os parâmetros de hemostasia. O objetivo da hemodiluição era o de manter o hematócrito em torno de $30 \%$. Esses resultados mostraram não haver interferência de hemodiluição, avaliada pela correlação entre hematócrito e os testes de coagulação no IO e POI, segundo Mezzano e $\mathrm{col}^{22}$, que mediram os níveis de albumina no PRÉ, IO e PO. Ao contrário do observado por Mammen e $\mathrm{col}^{2}$, a redução dos fatores de coagulação pareceu bem maior que a redução do hematócrito. Finalmente, o encurtamento do TLE demonstra ter ocorrido ativação da fibrinólise.

Dos testes realizados, a queda da contagem de plaquetas foi o único exame capaz de diferenciar os grupos com maior e menor sangramento para todos os tempos analisados. O grupo que sangrou em excesso apresentou redução de cerca de $77 \%$ dos níveis basais do PRÉ, enquanto que a redução foi em torno de $32 \%$ para o grupo que não sangrou em excesso no PO. Na nossa série, portanto, a influência maior foi deste parâmetro e poderíamos considerar a trombocitopenia como a causa principal dos distúrbios hemorrágicos.

O oxigenador de bolhas utilizado em todas as crianças poderia ser responsável por maior traumatismo dos elementos sangüíneos, incluindo as plaquetas, embora não possamos confirmar essa associação, pois não utilizamos outro tipo de oxigenador, como o de membrana ${ }^{5,23-25}$.

Outro fato importante foi a diferença significante do tempo de CEC entre os grupos com e sem sangramento excessivo $(\mathrm{p}<0,05)$. Além de trombocitopenia mais intensa, provavelmente, crianças com maior tempo de perfusão têm maior trauma nos elementos sanguiíneos, o que acaba levando a maior sangramento.

A incidência de hemorragia relacionada ao procedimento cirúrgico com CEC foi de $35 \%$. Esta taxaé mais elevada que a referida pela maioria dos autores, embora Wedemeyer e $\mathrm{col}^{26}$ tenham referido uma incidência de $40 \%$ em crianças com cardiopatia congênita cianogênica, que seriam mais susceptíveis a distúrbios de hemostasia.

A caracterização do grupo com maior sangramento é variável segundo esses autores. O sangramento acentuado por dreno de mediastino nas primeiras horas é o critério maior. Já o critério de reposição volêmica com hemoderivados não nos parece ideal. Acreditamos mais, que o conjunto de informações sobre o débito dos drenos, somado aos dados laboratoriais alterados e aos dados clínicos, sejam os que identificam o grupo de risco.

A hemorragia no PO é situação ainda mais grave em pacientes com evolução complicada. Em nossa série, quatro crianças apresentaram disfunção de múltiplos órgãos e sistemas, o que incluía uma disfunção hematológica, com mortalidade de 17,6\%. O óbito mais precoce (16h de PO) ocorreu com a criança que permaneceu mais tempo em CEC (130min) e as outras duas crianças que faleceram, também, apresentaram tempo de CEC prolongado (>105min), corroborando os dados da literatura de que, quanto maior o tempo de perfusão, maior o trauma aos elementos celulares. Salientamos, que, dos seis pacientes que sangraram excessivamente, todos apresentavam alteração em pelo menos um dos testes realizados no PRÉ.

Atribuímos, essencialmente, ao consumo dos fatores, as alterações ocorridas com a AP, fibrinogênio e fator V. Na nossa amostra não houve grande influência da hemodiluição. Para a AP, os nossos dados são concordantes em intensidade de redução com os de Schoeffel e col $^{3}$.

O prolongamento do TTPA durante a CEC reflete a ação da heparina no IO e, como ainda se fez presente no POI, não podemos descartar duas possibilidades: neutralização inadequada com as doses de protamina utilizadas ou rebote de heparina. Por certo, houve também consumo dos fatores de coagulação, mas a recuperação rápida dos níveis pré-operatórios, a partir do $1 \mathrm{PO}$ sugere que as alterações do TTPA sejam mais reflexo da ação da heparina, do que influenciadas por um processo de coagulação intravascular no POI. A análise comparativa entre pacientes que sangraram e aqueles que não tiveram sangramento excessivo não mostrou diferença significante entre os mesmos. Notou-se, entretanto, que para todos os tempos, as médias dos deltas percentuais do TTPA do grupo que não sangrou foram maiores que as médias do grupo que sangrou. As diferenças são aparentemente significativas no IO $(460 \%$ x $305 \%)$ e no $4-7 \mathrm{PO}(34 \%$ x 23\%). Isto pode ser devido à reposição de hemoderivados, que foi maior no grupo com sangramento. As crianças receberam mais plasma fresco e mais crioprecipitado, o que reduziria o valor do TTPA. Porém, esta diferença também existiu no IO durante a $\mathrm{CEC}$, refletindo, talvez, o fato de que o grupo que não sangrou tenha obtido níveis sangüíneos de heparina maiores que o grupo que sangrou e, com isso, ocorrendo menor ativação da coagulação e, conseqüentemente, menor sangramento.

No PO, a redução na contagem de plaquetas manteve-se até o $4^{\circ}$ dia, o que consideramos uma recuperação lenta, mas de acordo com os dados de literatura ${ }^{2,27-29}$. Há consenso entre os autores de que a cirurgia com CEC promove dano plaquetário de graus variáveis, de acordo com a temperatura utilizada e o tempo de perfusão. Estas alterações são conseqüência da ativação, que ocorre com a interface sangue-circuito e não são influenciadas pela hemodiluição, fato este defendido por Bick ${ }^{6}$. Dentre as inúmeras causas 
de sangramento no PO, a trombocitopenia e alterações qualitativas das plaquetas são consideradas como causas principais. A análise comparativa dos nossos dados revelou que este parâmetro, diferenciou os dois grupos, havendo redução, significantemente, maior na contagem de plaquetas no grupo que sangrou excessivamente.

A redução do fibrinogênio ocorrida no IO manteve-se no POI. A partir do 1PO houve aumento significante deste fator, sugerindo consumo pela ativação da coagulação no IO. Porém, a partir do $1 \mathrm{PO}$ o fibrinogênio comportou-se como uma proteína de fase aguda inflamatória. Boyd e $\mathrm{col}^{30}$ observaram aumento do fibrinogênio, mais tardio, em torno do $5^{\circ}$ dia, mas Douglas e col ${ }^{31}$ observaram tal aumento já a partir do $1^{\circ}$ dia.

No $P O$, a recuperação do fator $\mathrm{V}$ foi mais rápida que a da AP. Como ele não é dependente de vitamina K, pode-se inferir que não são apenas os fatores dependentes de vitamina K que estão alterados nesses pacientes.

No IO, houve um encurtamento significante do TLE durante a CEC, em consequiência de ativação da fibrinólise, porém, no POI já houve recuperação do TLE, exceto para uma criança que apresentou sangramento excessivo. Não se observou, portanto, hiperfibrinólise no PO.

Pôde-se observar ainda redução significante do hematócrito no IO, conseqüência do processo de hemo- diluição, a que foram submetidas todas as crianças. Houve recuperação imediata conseqüente a reposição de concentrado de hemácias e a reversão da hemodiluição. Ao contrário de alguns autores, não pudemos atribuir à hemodiluição as alterações dos fatores de coagulação e trombocitopenia encontradas no $\mathrm{IO}$ e $\mathrm{POI}^{2-4}$.

As alterações da hemostasia ocorridas no IO são consequiências do trauma sofrido pelos elementos sangüíneos no circuito extracorpóreo, desencadeando-se assim um processo de ativação da coagulação e do processo fibrinolítico. Houve alterações significantes da AP, do fibrinogênio, das plaquetas, do fator V e do TLE. As complicações hemorrágicas relacionadas a distúrbios de coagulação apresentaram incidência elevada (35\%), especulando-se se, eventualmente, a alta prevalência de desnutrição estaria associada a estas complicações. O uso de oxigenador de bolhas poderia, também, ser responsável pela alta prevalência de complicações hemorrágicas nesses pacientes. As crianças que apresentaram sangramento excessivo foram aquelas com maior tempo de perfusão. A contagem de plaquetas no $\mathrm{IO}$ foi o único parâmetro capaz de diferenciar os grupos com maior e menor sangramento no POI, sendo que crianças com algum distúrbio da hemostasia no PRÉ tiveram maior probabilidade de hemorragia, quando submetidos à cirurgia cardíaca com CEC.

\section{Referências}

1. Weiland AP, Walker WE - Physiologic principles and clinical sequelae of cardiopulmonary bypass. Heart Lung 1986; 15: 34-9.

2. Mammen EF, Koets MH, Washington BC et al - Hemostasis changes during cardiopulmonary bypass surgery. Semin Thromb Hemost 1985; 11: 281-92.

3. Schoeffel D, Schimpf K, Krier C-Selected blood coagulation parameters during extracorporeal circulation. Behring Inst Meit 1986; 79: 104-11.

4. Bagge I, Lilienberg G, Nystrom SO, Tydén H - Coagulation, fibrinolysis and bleeding after open-heart surgery. Scand J Thorac Cardiovasc Surg 1986; 20: 151-60.

5. Perloff JK, Rosove MH, Child JS, Wright GB - Adults with cyanotic congenital heart disease: Hematologic management. Ann Intern Med 1988; 109: 406-13.

6. Bick RL - Hemostasis defects associated with cardiac surgery, prosthetic devices, and other extracorporeal circuits. Semin Thromb Hemost 1985; 11: 24979.

7. Ware JA, Reaves K, Horak JK, Solis T-Defective platelet aggregation in patients undergoing surgical repair of cyanotic congenital heart disease. Ann Thorac Surg 1983; 36: 289-94.

8. Kongsgaard UE, Smith-Erichsen N, Geiran O, Bjornskau I - Changes in the coagulation and fibrinolytic systems during and after cardiopulmonary bypass surgery. Thorac Cardioavasc Surgeon 1989; 37: 158-62.

9. GomezF, Galvan RR, Freuk S, MunozJC, Chavez R, Vásquez J - Mortality in second and and third degree malnutrition. J Trop Pediatric 1956; 2: 77-85.

10. Waterlow JC - Classification and definition of protein-calorie malnutrition. $\mathrm{Br}$ Med J 1972; 3:566-9.

11. Maluf MA - Protocolo de circulação extracorpórea para crianças. In: Andrade JCS, ed - Circulação Extracorpórea. São Paulo: Angelus, 1988: 89-110.

12. Quick AJ, Stanley-Brown M, Bancroft FW - A study of the coagulation defect in hemophilia and in jaundice. Am J Med Sci 1935; 190: 601-11.

13. Proctor PR, Rapaport SI - The partial thromboplastin time with kaolin: a simple screening test for first stage plasma clotting factor deficiencies. Am J Clin Pathol 1961; 36: 212-9.

14. Brecher G, Cronkite MP - Morphology and enumeration of human blood platelets. J Appl Physiol 1950; 3: 365-77.

15. Quick AJ - The assay and properties of labile factor V. J Clin Pathol 1960; 13:45761 .

16. Clauss A - Gerinnungs physiologische schnell methode zur bestimumg des fibrinogens. Acta Haematol 1957; 17: 237-46.

17. Kluft C-Quantitation and behavior of extrinsic or vascular plasminogen activa- tor in blood. In: Progress in Fibrinolysis. Vol V. Churchill Livingstone, 1981: 24-30.

18. Siegel S - Estatistica no Parametrica. México: Trillas, 1975: 346.

19. Leite HP, Fisberg M, Novo NF, Nogueira EB, Ueda IK - Nutritional assessment and surgical risk markers in children submitted to cardiac surgery. Rev Paul Med 1995; 113: 706-14.

20. Komp DM, Sparrow AW - Polycythemia in cyanotic heart disease - A study of altered coagulation. Pediatrics 1970; 76: 231-6.

21. Colón-Otero G, Gilchrist GS, Holcomb GR, Ilstrup DM, Bowie EJW - Preoperative evaluation of hemostasis in patients with congenital heart disease. Mayo Clin Proc 1987; 62: 379-85.

22. Mezzano D, Habash J, Aranda E et al - Consumo de plaquetas y disfunción plaquetaria durante y después de la cirurgia con circulation extracorporea. Sangre 1986; 31: 425-34.

23. Oeveren vanW, Kazatchkine MD, Descamps-Latscha B et al - A prospective study of bubble versus membrane oxigenation. J Thorac Cardiovasc Surg 1985; 89: 888-99.

24. Boonstra PW, Vermeulen FE, Leusink JA et al - Hematological advantage of membrane oxygenator over a bubble oxygenator in long perfusions. Ann Thorac Surg 1986; 41: 297-300.

25. Gomes WJ - Tipos de oxigenadores. In: Andrade JCS, eds - Circulação Extracorpórea. São Paulo: Angelus, 1988: 51-5.

26. Wedemeyer AL, Castaneda AR, Edson JR, Krivit W - Serial coagulation studies in patients undergoing Mustard procedure. Ann Thorac Surg 1973; 15: 120-7.

27. BachmannF,McKennaR,ColeER,NajafiH-The hemostatic mechanismafteropenheart surgery. I. Studies on plasma coagulation factors and fibrinolysis in $512 \mathrm{pa}-$ tients after extracorporeal circulation. J Thorac Cardiovasc Surg 1975; 70: 76-85.

28. Schmidt PJ, Peden JC, Brecher G, Baranovsky A - Thrombocytopenia and bleeding tendency after extracorporeal circulation. N Eng J Med 1961; 265: 1181-4.

29. Holloway DS, Summaria L, Sandesara J, Vagher JP, Alexander JC, Caprini JA Decreased platelet number and function and increased fibrinolysis contribute to postoperative bleeding in cardiopuimonary bypass patients. Thromb Haemost 1988; 59: 62-7.

30. Boyd AD, Engelnan RM, Beaudet RL, Lackner H - Disseminated intravascular coagulation following extracorporeal circulation. J Thorac Cardiovasc Surg 1972; 64: 685-93.

31. Douglas AS, McNicol GP, Bain WH, Mackley WA - The haemostatic defect following extracorporeal circulation. Br J Surg 1966; 53: 455-7. 